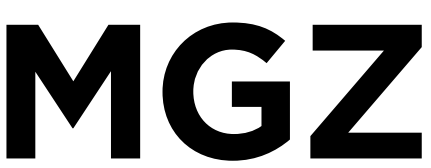

\title{
MILITÄRGESCHICHTLICHE ZEITSCHRIFT
}

\section{BEGRÜNDET VOM}

Militärgeschichtlichen Forschungsamt

HERAUSGEGEBEN VOM

Zentrum für Militärgeschichte und

Sozialwissenschaften der Bundeswehr

DURCH

Hans-Hubertus Mack

Michael Epkenhans

IN VERBINDUNG MIT

Katherine B. Aaslestad

Ute Daniel

Stig Förster

Bernhard R. Kroener

Wilfried Loth

Reiner Pommerin

Sir Hew Strachan

Bernd Wegner

\section{REDAKTION}

Gabriele Bosch

Jörg Echternkamp

Dorothee Hochstetter

Dieter H. Kollmer

Thorsten Loch

Markus Pöhlmann

Aleksandar-S. Vuletić

John Zimmermann

\section{DE GRUYTER OLDENBOURG}


ABSTRACTED/INDEXED IN Baidu Scholar; Celdes, CNKI Scholar (China National Knowledge Infrastructure), CNPIEC, De Gruyter - IBR (International Bibliography of Reviews of Scholarly Literature in the Humanities and Social Sciences), - IBZ (International Bibliography of Periodical Literature in the Humanities and Social Sciences), EBSCO - Historical Abstracts, - TOC Premier, - Discovery Service, Elsevier - SCOPUS, Google Scholar, J-Gate, JournalTOCs, L‘Année philologique, Naviga (Softweco), Primo Central (ExLibris), ReadCube, ResearchGate, SCImago (SJR), Summon (Serials Solutions/ProQuest), TDOne (TDNet), Thomson Reuters - Arts \& Humanities Citation Index, - Current Contents/Arts \& Humanities, WorldCat (OCLC)

ISSN 2193-2336 · e-ISSN 2196-6850

Texte zur Erstveröffentlichung bitte als Datei (MS Word) und als Ausdruck zusenden. Nach Annahme des Manuskripts wird die angelieferte Datei zur alleinigen Grundlage des Lektorats. Erwünschte Änderungen sind auf der Korrekturfahne vorzunehmen. Formale Hinweise zur Manuskriptgestaltung finden Sie auf der Homepage des ZMSBw (www.zmsbw.de).

Beiträge in dieser Zeitschrift geben jeweils die persönliche Meinung des Verfassers wieder. Irrtum vorbehalten bei Angabe der Preise und der ISBN von besprochenen Publikationen. Alle Informationen zur Zeitschrift, wie Hinweise für Autoren, Open Access, Bezugsbedingungen und Bestellformulare, sind online zu finden unter www.degruyter.com/mgzs

VERANTWORTLICHE HERAUSGEBER Hans-Hubertus Mack und Michael Epkenhans (ZMSBw)

REDAKTION Zentrum für Militärgeschichte und Sozialwissenschaften der Bundeswehr (ZMSBw) Redaktion MGZ, Postfach 6011 22, 14411 Potsdam, E-Mail: mgzredaktion@bundeswehr.org

JOURNAL MANAGER Gabriele Jaroschka, De Gruyter, Rosenheimer Straße 143, 81671 München, Deutschland, Tel.: +49 (0)8976 902-443, E-Mail: Gabriele.Jaroschka@ degruyter.com

ANZEIGENVERANTWORTLICHER Heiko Schulze, De Gruyter, Genthiner Straße 13, 10785 Berlin, Deutschland, Tel.: +49 (0)30 260 05-358, Fax: +49 (0)30 260 05-264

E-Mail: anzeigen@degruyter.com

LEKTORAT Esther Geiger

SATZ jürgen ullrich typosatz, Nördlingen

DRUCK Franz X. Stückle Druck und Verlag e.K., Ettenheim

Printed in Germany

(C) 2017 ZMSBw, Zeppelinstr. 127/128, 14471 Potsdam, Tel.: +49 (0)331 9714-0, Fax: -509; E-Mail: zmsbweingang@bundeswehr.org

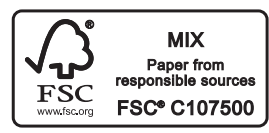




\section{Inhalt}

Editorial -1

\section{Zur Geschichte der MGM/MGZ}

Jörg Echternkamp

Mittler der Militärgeschichte: "Militärgeschichtliche Mitteilungen«.

Wissenschafts- und mediengeschichtliche Anmerkungen zur Entstehung und Entwicklung einer »amtlichen« Fachzeitschrift — 12

Klaus A. Maier

Persönliche Erinnerungen an eine 20-jährige Redaktionstätigkeit

für die »Militärgeschichtlichen Mitteilungen» — 64

Hans-Joachim Beth

Die »Militärgeschichtlichen Mitteilungen« im Blick der ostdeutschen „Militärgeschichte«. Persönliche Eindrücke eines Redakteurs — 68

\section{MGM/MGZ wiedergelesen}

\section{Reiner Pommerin}

Standortbestimmung am Fuß einer Leiter. Rainer Wohlfeil,

Wehr-, Kriegs- oder Militärgeschichte? (MGM, 1, 1967, 1) — 72

Gerd Krumeich

Ludendorffs Dolchstoß. Eberhard Kessel, Ludendorffs

Waffenstillstandsforderung vom 29. September 1918 (MGM, 4, 1968, 1)

Klaus Naumann

Militär - Politik - Professionalismus. Michael Geyer, „Die Wehrmacht der deutschen Republik ist die Reichswehr«. Bemerkungen zur neueren Literatur (MGM, 14, 1973, 2) - 91 
Johann Chapoutot

Das Deutsche Kaiserreich am Vorabend des Ersten Weltkrieges - ein »unregierbares Gebilde«? Wolfgang J. Mommsen, Die latente Krise des wilhelminischen Reiches. Staat und Gesellschaft in Deutschland 1890-1914 (MGM, $15,1974,1)-99$

Holger H. Herwig

Clio und die Marine. Gerhard Schreiber, Zur Kontinuität des Groß- und Weltmachtstrebens der deutschen Marineführung (MGM, 26, 1979, 2) - 105

\section{Roger Chickering}

Militarismus und Bellizismus. Detlef Vogel, Militarismus - unzeitgemäßer Begriff oder modernes historisches Hilfsmittel? Zur Militarismuskritik im 19. und 20. Jahrhundert in Deutschland (MGM, 39, 1986, 1) - 110

\section{Johannes Hürter}

Die Reichswehr in der Republik. Wilhelm Deist, Die Reichswehr und der Krieg der Zukunft (MGM, 45, 1989, 1) - 120

\section{Wilfried Loth}

Die USA und die Stalin-Note vom 10. März 1952. Reinhard Neebe, Wahlen als Test: Eine gescheiterte Initiative des Politischen Planungsstabs im State Department zur Stalin-Note vom 10. März 1952 (MGM, 45, 1989, 1) — 126

Bernd Wegner

Weltkrieg 1938? Ivan Pfaff, Die Modalitäten der Verteidigung der Tschechoslowakei 1938 ohne Verbündete (MGM, 57, 1998, 1) — 133

Katherine B. Aaslestad

New Military History and The Napoleonic Wars. Karen Hagemann, Tod für das Vaterland: Der patriotisch-nationale Heldenkult zur Zeit der Freiheitskriege (MGZ, 60, 2001, 2) - 146

\section{Stig Förster}

Der Streit um den Schlieffenplan. Terence M. Holmes, Der Schlieffenplan des Friedrich von Bernhardi: Zur Beilegung eines mythischen Streitfalls (MGZ, 63, 2004, 2) - 152 
Sönke Neitzel

Ein neues Kapitel der Zeitgeschichte. Bernhard Chiari, Die Bundeswehr als Zauberlehrling der Politik? Der ISAF-Einsatz und das Provincial Reconstruction Team (PRT) Kunduz 2003 bis 2012 (MGZ, 72, 2013, 2) — 160

Dennis E. Showalter

A Bavarian Prince and a Soldier of Germany. Holger Afflerbach, Kronprinz Rupprecht von Bayern im Ersten Weltkrieg (MGZ, 75, 2016, 1) — 168

Karen Hagemann

Militär, Krieg und Geschlecht: Ein Kommentar zur Militärgeschichtsschreibung in der MGZ - 175

\section{Gesamtregister der MGM/MGZ 1967-2016}

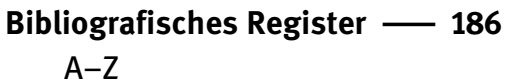

Sachregister: Epochen — 219

Militärgeschichte vor 1789; Militärgeschichte 1789-1913; Zeitalter der Weltkriege; Deutsche Militärgeschichte nach 1945; Neueste Militärgeschichte ab 1990

\section{Sachregister: Geografische Räume — 251}

Europäische Militärgeschichte; Außereuropäische Militärgeschichte; Globalgeschichte

\section{Sachregister: Themenfelder und Schlagworte - 263}

Antisemitismus; Auslandseinsätze der Bundeswehr; Befestigungswesen und Architektur; Biografisches; Desertion; Deutsche Teilung und Wiedervereinigung; Diplomatie und Internationale Politik; Erfahrungswelten und Identitäten; Erinnerungskultur; Erziehung und (Aus-)Bildung; Gender; Institutionen; Kindheit, Jugend und Schule; Kolonien und Kolonialpolitik; Kriegführung; Kriegsgefangenschaft; Kriegstheorie und Kriegsbilder; Kriegs- und Rüstungswirtschaft; Kriegsverbrechen; Medien; Medizin und Sanitätswesen; Methodik, Theorie und Historiografie der (Militär-)Geschichtsschreibung; Militärische Operationen und Schlachten; Militärische Planungen und Kriegsziele; Militärische und paramilitärische Organisationen; Militärische und zivile Opfer; Militärreformen; Militärsoziologie; Militär 
und Gesellschaft; Militär und Religion; Nachrichtendienste und Spionage; Nachschub und Logistik; Okkupationspolitik und Besatzung; Ost-West-Konflikt; Personalwesen und Personalpolitik; Politik und Parteien; Politische Ideen und Ideologien; Propaganda und Zensur; Recht; Rüstung und Abrüstung; Soziale Versorgung und Truppenbetreuung; Sport; Strategie und Taktik; Teilstreitkräfte; Tradition und Rituale; Vereine, Verbände und Stiftungen; Verwundung und Leiden im Krieg; Völkermord; Wehrorganisation und -verwaltung; Widerstand gegen den Nationalsozialismus; Wiederbewaffnung

Mitarbeiterinnen und Mitarbeiter — 332 